\title{
Mosaicity, dislocations and strain in heteroepitaxial diamond grown on iridium
}

\author{
H. Bensalah ${ }^{\mathrm{a}}$, I. Stenger ${ }^{\mathrm{a}}$, G.Sakr ${ }^{\mathrm{a}}$, J.Barjon ${ }^{\mathrm{a}}$, R.Bachelet ${ }^{\mathrm{b}}$, A.Tallaire ${ }^{\mathrm{c}}$, J. Achard ${ }^{\mathrm{c}}$, N. Vaissiere $^{\mathrm{d}}$, K.H.Lee ${ }^{\mathrm{d}}$, \\ S. Saada ${ }^{\mathrm{d}}$, J.C.Arnault ${ }^{\mathrm{d}}$
a Groupe d'Etude de la Matière Condensée (GEMaC), Université de Versailles St Quentin en Yvelines, CNRS, Université Paris Saclay, 45 Avenue des Etats-Unis, 78035 Versailles, France b Université de Lyon, Ecole Centrale de Lyon, INL-UMR5270-CNRS, Ecully, France
c Paris 13, Sorbonne Paris Cité, Laboratoire des Sciences des Procédés et des Matériaux (CNRS UPR 3407), Villetaneuse, France
d CEA, LIST, Diamond Sensors Laboratory, Gif sur Yvette, France

\begin{abstract}
The present study provides a multi-scale investigation of the crystalline quality and the structural defects present in heteroepitaxial diamond films grown on iridium $/ \mathrm{SrTiO}_{3}(001)$ substrates by microwave plasma assisted chemical vapor deposition. X-ray diffraction, Raman spectroscopy and low temperature cathodoluminescence are combined to accurately characterize the mosaicity, the density of dislocations and the residual strain within the films. X-ray diffraction and Raman results confirm a structural quality at the state-of-the-art according to the epitaxial relationship $\langle 100\rangle$ diamond(001) // $\langle 100\rangle$ iridium(001) // $\langle 100\rangle \mathrm{SrTiO}_{3}(001)$. In addition, Raman and cathodoluminescence observations on cross-sections reveal the presence of local strain.
\end{abstract}

\section{Introduction}

Promising advances have been recently reported for the growth of diamond single crystals on large areas [1]. Cloning and tiling of diamond single crystals allow enlarging lateral dimensions of homoepitaxial diamond films [2]. At the same time, heteroepitaxial diamond constitutes a credible alternative to homoepitaxy for the next generation of power electronic or radiation detection applications. Indeed, the upscaling perspectives for the growth process are particularly interesting for producing large diamond surfaces, compatible with microelectronic technologies [3]. Among the potential substrates, iridium is up to now the best candidate to heteroepitaxially grow high quality diamond by the Chemical Vapor Deposition (CVD) technique. This behavior is related to a specific reactivity of iridium surface towards active species during Bias Enhanced Nucleation (BEN) [4] compared to other substrates like silicon [5] or silicon carbide [6,7]. After BEN, surface investigations revealed diamond nucleation within specific areas called domains [8]. Contrary to other substrates, growth of diamond nuclei is prevented at this stage. Iridium epitaxial layers are currently deposited

\footnotetext{
* Corresponding author.

E-mail address: julien.barjon@uvsq.fr (J. Barjon).
}

onto single crystal $\mathrm{MgO}, \mathrm{SrTiO}_{3}$ or up-scalable substrates like YSZ (Yttria-stabilized zirconia)-buffered on silicon (001) [9].

However, the crystalline quality of heteroepitaxial diamond grown on iridium is still limited by some important problems such as the residual mosaicity, the residual strain and the presence of a high density of dislocations. For a quantitative evaluation of dislocation density in heteroepitaxial diamond, $C$. Stehl et al. have used chemical etching and they obtained values in the range of $7 \times 10^{9}-5 \times 10^{7} \mathrm{~cm}^{-2}$ for 10 and $1000 \mu \mathrm{m}$ diamond thicknesses, respectively [10]. While using $\mu$ Raman spectroscopy and X- ray diffraction, M. Fisher et al. have demonstrated that stress up to several GPa can be generated in diamond grown on iridium [11]. These problems can affect the electron-hole mobilitylifetime product and the charge collection efficiency of detectors [12]. In fact, a careful control of the growth conditions is required to decrease the density of defects and then improve the quality of diamond films.

The objective of the present work is to provide a better understanding of the specific defects like dislocations and strain in heteroepitaxial diamond films grown on iridium $/ \mathrm{SrTiO}_{3}$ (001). The analysis is carried out by combining different techniques including Raman, X- ray diffraction and cathodoluminescence (CL) at $10 \mathrm{~K}$ using a multi-scale approach. Cross-sections of samples were also prepared to follow the dislocation propagation along the growth axis. 


\section{Experimental details}

Diamond thin films were heteroepitaxially grown on $\mathrm{Ir} / \mathrm{SrTiO}_{3}(001)$ substrates by microwave plasma assisted chemical vapor deposition (MPCVD). First, iridium layers (200 $\mathrm{nm}$ ) were deposited by e-beam evaporation under secondary vacuum $\left(<10^{-6}\right.$ mbar $)$ on $5 \times 5 \mathrm{~mm}^{2}$ (001) $\mathrm{SrTiO}_{3}$ single crystals purchased from CrysTec Inc. The sample temperature was measured by an infrared pyrometer with a precision of $0.3 \%$ on a $\mathrm{SiO}_{2}$ wafer placed next to $\mathrm{SrTiO}_{3}$ substrates. The iridium growth rate was $1.5 \mathrm{~nm} / \mathrm{min}$ and the substrate temperature was $980{ }^{\circ} \mathrm{C}$. The polar and azimuthal mosaicity of these layers were measured by X-ray diffraction and can be as low as $0.14^{\circ}$ and $0.12^{\circ}$, respectively.

To induce diamond epitaxial nucleation on Ir, a Bias Enhanced Nucleation (BEN) procedure was applied. The experimental conditions are provided in Table 1 . A negative bias voltage of $-307 \mathrm{~V}$ was applied to the substrate. The BEN step was followed by a first growth step of $60 \mathrm{~nm}$ performed in the same MPCVD reactor [8]. A second growth step was necessary to achieve thick diamond films in the order of $300 \mu \mathrm{m}$ thickness, by using the high growth rate $(6 \mu \mathrm{m} / \mathrm{h})$ conditions of homoepitaxial growth well-mastered at LSPM [23]. This was carried out in a bell-jar type reactor (Plassys BJS150). No nitrogen was intentionally added to the gas phase. Four samples were obtained by varying the second growth step duration: 0 (no second growth step), 18, 30 and $48 \mathrm{~h}$. At the end, the diamond layer thicknesses are: $60 \mathrm{~nm}, 90 \mu \mathrm{m}$, $200 \mu \mathrm{m}$ and $320 \mu \mathrm{m}$, respectively.

Different techniques have been carried out to analyze the structural and physical properties of heteroepitaxial diamond samples. Conventional X-ray diffraction (XRD) gives a characterization of structural properties averaged over the crystal volume. On the contrary, Raman or $\mathrm{CL}$ are using focused beam and are then sensitive to a typical $\mu \mathrm{m}$ depth region. In order to understand the evolution of the heteroepitaxial diamond properties along the growth axis, heteroepitaxial diamond were characterized on the final growth surface but also on their cross-sections. For preparing cross-sections, the samples were cut by a Nd:YAG laser along the vertical (100) plane and then the edge was polished using argon ion beam in the CrossPolisher JEOL IB- 09010CP at a voltage $4 \mathrm{kV}$ during $10 \mathrm{~h}$.

Confocal Raman spectroscopy and mapping of the $1332 \mathrm{~cm}^{-1}$ diamond line position and full-width-at-half maximum (FWHM) were performed on the samples. The data were recorded in a backscattering geometry using a Horiba Jobin Yvon confocal micro-Raman HR800. Excitation was performed with the $514 \mathrm{~nm}$ line of $\mathrm{Ar}^{+}$laser, focused onto the sample with an $\times 100$ objective. A $632 \mathrm{~nm}$ He-Ne laser was also used in the same conditions to compare our data to the literature. By using an 1800 grooves $/ \mathrm{mm}$ grating and a confocal hole of $50 \mu \mathrm{m}$, the spectral resolution was $0.9 \mathrm{~cm}^{-1}$ in the range of the diamond peak, while the interpixel distance equals to $0.48 \mathrm{~cm}^{-1}$. The Raman mappings were recorded every $1 \mu \mathrm{m}$ step thanks to a motor-driven XY table. Each spectrum was automatically fitted using a Lorentzian function in order to

Table 1

Experimental conditions of BEN and CVD growth steps applied to $\mathrm{Ir} / \mathrm{SrTiO}_{3}(001)$ substrates.

\begin{tabular}{|c|c|c|c|c|c|}
\hline & \multicolumn{3}{|c|}{ Nucleation process } & \multirow[t]{2}{*}{ Growth1 } & \multirow[t]{2}{*}{ Growth2 } \\
\hline & $\begin{array}{l}\mathrm{H}_{2} \\
\text { cleaning }\end{array}$ & $\begin{array}{l}\mathrm{H}_{2} / \mathrm{CH}_{4} \\
\text { stabilization }\end{array}$ & BEN & & \\
\hline $\mathrm{CH}_{4}(\%)$ & 0 & 4 & 4 & 0.6 & 5 \\
\hline Pressure (mbar) & 20 & 20 & 20 & 20 & 200 \\
\hline MW Power (W) & 400 & 400 & 400 & 400 & 3000 \\
\hline Bias voltage (V) & 0 & 0 & -307 & 0 & 0 \\
\hline $\begin{array}{l}\text { Substrate } \\
\text { temperature }\left({ }^{\circ} \mathrm{C}\right)\end{array}$ & $\begin{array}{l}600 \pm \\
50\end{array}$ & $650 \pm 50$ & $\begin{array}{l}700 \pm \\
50\end{array}$ & $\begin{array}{l}600 \pm \\
50\end{array}$ & $880 \pm 30$ \\
\hline Duration (min) & 10 & 10 & $40-45$ & 30 & $\begin{array}{l}0,18,30 \text { and } \\
48 \mathrm{~h}\end{array}$ \\
\hline
\end{tabular}

extract position and FWHM of the Raman line, further color-plotted in two-dimension graphs.

The structural properties of diamond films were investigated by Xray diffraction. A Rigaku SmartLab diffractometer equipped with a high brilliance rotating anode and an in-plane arm (allowing grazing incidence XRD) was used. Conventional $\theta / 2 \theta$ scan and $\omega$-scan (rocking curve) were done on the symmetric reflection (004) to analyze the out-of-plane lattice parameter and the polar mosaicity (out-of-plane), respectively. The azimuthal mosaicity (in-plane) is evaluated by recording azimuthal scans ( $\phi$-scans) on asymmetric reflections. Reciprocal space maps have been further recorded around the symmetric (004) and asymmetric $(-1-13)$ nodes to characterize the mosaicity and the possible residual strain. The azimuthal mosaicity and the microstrain level at the diamond surface has been also probed by surface diffraction in grazing incidence (GIXRD) conditions on (220) reflection (with fixed incidence of $0.35^{\circ}$ and lateral soller slits of $0.114^{\circ}$ ).

CL spectra were collected using an optical detection system from Horiba Jobin Yvon SA, installed on a JEOL7001F field-emission scanning electron microscope (SEM). The samples were mounted on a GATAN cryostat SEM-stage, which was cooled at $10 \mathrm{~K}$ with liquid helium. The specimens were coated with a semitransparent gold layer of $5 \mathrm{~nm}$ in order to evacuate away electrical charges. The penetration depth of electrons in diamond is $0.9 \mu \mathrm{m}$ depth with the $10 \mathrm{keV}$ electrons used here. The CL emission was collected by a parabolic mirror and focused with mirror optics on the entrance slit of a $55 \mathrm{~cm}$ focal length monochromator. This achromatic all-mirror optics bench, combined with a suitable choice of ultraviolet (UV) detectors and gratings, provides an excellent sensitivity down to $200 \mathrm{~nm}$. A silicon charge-coupleddisplay camera was used to record spectra. Monochromatic CL images were taken by filtering the excitonic signal through the monochromator equipped with a photomultiplier detector synchronized with the beam scanning.

\section{Results and discussion}

\subsection{Raman spectroscopy as a function of the film thickness}

An optical image of the $320 \mu \mathrm{m}$ thick heteroepitaxial diamond crystal is presented in Fig. 1. The diamond film is still standing on the $5 \times 5 \mathrm{~mm}^{2} \mathrm{Ir} / \mathrm{SrTiO}_{3}(001)$ substrate, which indicates that the residual strain of diamond film is low enough to avoid its spontaneous

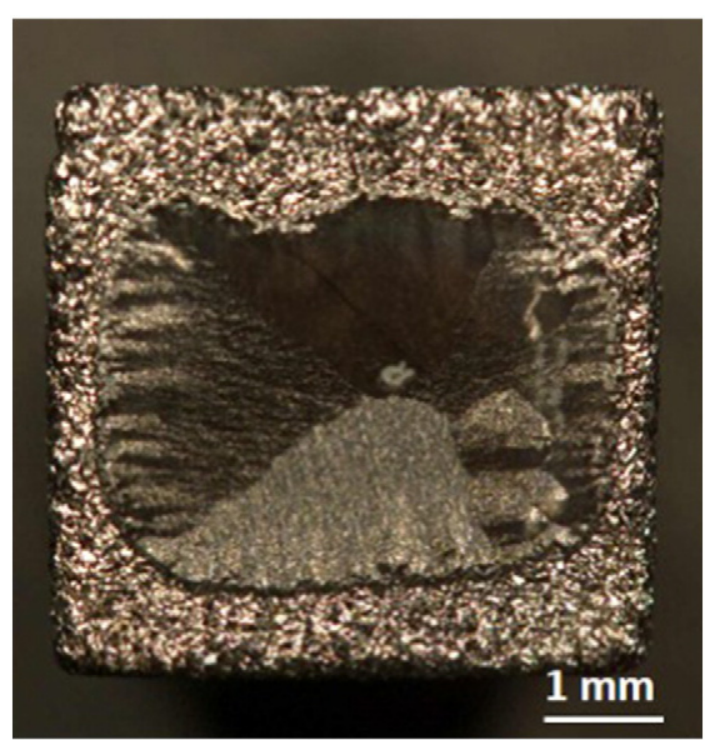

Fig. 1. Optical image of a $320 \mu \mathrm{m}$ thick heteroepitaxial diamond film grown on $\mathrm{Ir} / \mathrm{SrTiO}_{3}$ (001) substrate. 
separation from its substrate (delamination), as frequently reported in the literature [9]. The central part was single crystalline $\left(4 \times 3 \mathrm{~mm}^{2}\right)$, while we observe the formation of polycrystalline diamond close to the borders. The formation of polycrystalline diamond is correlated to SEM observations recorded just after the BEN process (not shown here) which reveal the absence of heteroepitaxial domains at the borders [8].

Raman results of the different samples are summarized in Fig. 2. They were obtained from averaging spectra recorded at 5 different locations of the final growth surface. The FWHM of first order diamond peak $\left(1332 \mathrm{~cm}^{-1}\right)$ is commonly used as an indicator of the structural disorder. The narrower the FWHM, the better the structural quality. We observe clearly from the plot that with increasing film thickness, the Raman FWHM decreases. The linewidth of heteroepitaxial diamond then get closer to the FWHM value obtained for a reference "electronic grade" single crystal CVD diamond (Element 6 ). This means that the diamond structural quality improves with thickness. It confirms the recent observations of C. Stehl et al. [10].

Comparative measurements of Raman linewidths with literature require that the same excitation laser is used, as the intrinsic FWHM depends on the excitation wavelength. Therefore the 200 and $320 \mu \mathrm{m}$ thick samples were also measured with a He-Ne laser at $632 \mathrm{~nm}$. We found linewidths of $2.1 \pm 0.2$ and $2.2 \pm 0.2 \mathrm{~cm}^{-1}$ respectively. Authors of ref. [10] report FWHM of 4 and $2.5 \mathrm{~cm}^{-1}$ with the same $632 \mathrm{~nm}$ excitation at thicknesses of 100 and $300 \mu \mathrm{m}$ respectively. The Raman linewidths of heteroepitaxial diamond crystal presented here are slightly narrower for comparable thicknesses. This leads to first conclude that the heteroepitaxial diamond samples grown in this study present a structural quality at the current state-of-the-art.

\subsection{Structural analysis by XRD}

XRD characterizations were performed to study the thicker heteroepitaxial diamond film grown on $\mathrm{Ir} / \mathrm{SrTiO}_{3}(001)$. A symmetrical $\theta / 2 \theta$ scan performed on the center of the sample is presented in Fig. 3 (a). Only (001) reflections are observed which indicates that the diamond film and the iridium layer are both (001)-oriented. A tilt angle was however observed between the diamond film and the $\mathrm{SrTiO}_{3}$ substrate, that can be up to $\sim 1^{\circ}$ with respect to the $\langle 100\rangle$ direction of the substrate. The out-of-plane crystal mosaicity (polar orientation distribution) of the diamond film is measured by $\omega$-scan, also called rocking curve (Fig. 3(b)). The full width at half maximum (FWHM) of this curve, quantifying the mosaicity, is about $0.6^{\circ}$, in the same order of magnitude than other works on heteroepitaxial diamond for comparable thicknesses [14,15]. A large $\phi$-scan (azimuthal scan) was acquired on the asymmetric $\langle 202\rangle$ reflections (Fig. 3(c)) to establish the in-plane

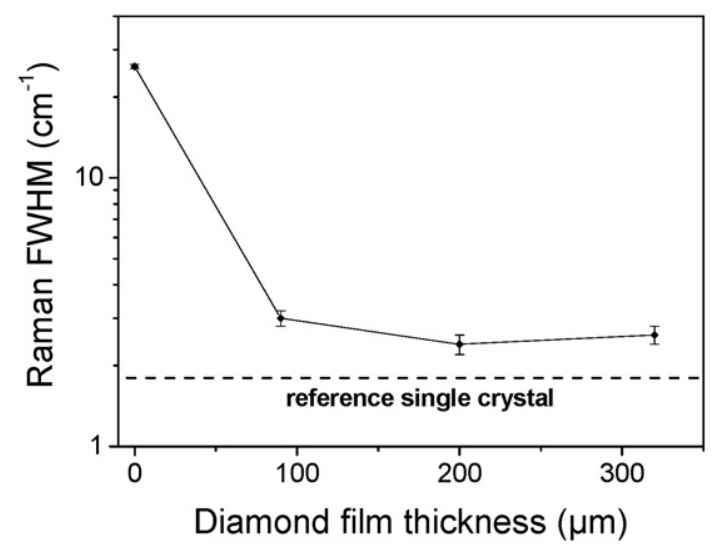

Fig. 2. Average FWHM of Raman first order diamond peak measured at the surface of heteroepitaxial diamond films of different thicknesses $(60 \mathrm{~nm}, 90 \mu \mathrm{m}, 200 \mu \mathrm{m}$ and $320 \mu \mathrm{m})$. The measurements were done with a $514 \mathrm{~nm}$ laser excitation. The reference single crystal value was taken from an electronic grade diamond from Element6. orientation of the diamond film and its epitaxial relationship with the substrate. Reflections appear every $90^{\circ}$, indicating that the diamond film is epitaxial with only one in-plane orientation. As previously reported, it is confirmed that diamond has been epitaxially grown cubeon-cube on $\mathrm{Ir} / \mathrm{SrTiO}_{3}(001)$ substrate and the epitaxial relationship can be written as follows: $\langle 100\rangle$ diamond $(001) / /\langle 100\rangle \operatorname{Ir}(001) / /\langle 100\rangle$ $\mathrm{SrTiO}_{3}(001)$. A zoom on one peak of this $\phi$-scan is presented on Fig. 3 (d). The FWHM of this curve gives the azimuthal mosaicity (in-plane orientation distribution, or so called twist, of the crystals), and it is again $<1^{\circ}$, close to $0.7^{\circ}$, confirming the good epitaxial quality of the diamond film.

A detailed investigation of the diamond (004) reflection in the $\theta / 2 \theta$ scan shows an asymmetric peak with a residual diffracted intensity at high angles (Fig. 4). In addition to the main peak for which the angular position of the maximum precisely corresponds to a fully relaxed diamond with a bulk lattice parameter $c=3.567 \AA$, a weaker and broader peak is also observed at higher angles. It reveals an out-of-plane deformation of the crystal lattice, and indicates that a small part of the diamond is strained with a smaller out-of-plane lattice parameter around $c=3.55 \AA$ ( $~ 0.5 \%$ strained $)$. In this conventional XRD geometry, the whole crystal is probed. Then, from the integrated intensities of the two peaks deconvoluted by fitting with pseudo-Voigt function, we can conclude that $\sim 97 \%$ of the diamond film is fully relaxed while the remaining $\sim 3 \%$ exhibit a smaller $c$ parameter. This can be explained by the in-plane tensile strain generated by heteroepitaxial growth on Ir ( $\mathrm{a}=3.84 \AA$ ) with a lattice mismatch of $+7.7 \%$, even if dislocations must accommodate this high structural mismatch.

In order to confirm this observation and further characterize the diamond film in more details, reciprocal space maps (RSM) have been recorded around its symmetrical (004) node (Fig. 5(a)). We observe that the main intensity of the node is located at $\mathrm{q}_{\mathrm{z}}=1.121 \AA^{-1}$, corresponding to the bulk cell parameter. This confirms that the main out-of-plane parameter corresponds to the bulk value ( $c=3.567 \AA$ ). The $(004)$ node exhibits a drag of residual diffracted intensity at high $\mathrm{q}_{z}$ values, corresponding to smaller out-of-plane lattice parameter than bulk value. This confirms that a small part of the diamond film is strained. Also, the node is elongated along the $\mathrm{q}_{\mathrm{x}}$ direction as a consequence of the mosaicity of the single-crystal. Such a feature is typical of the columnar growth of crystals obtained by heteroepitaxy on highly mismatched or mosaic substrates, such as $\mathrm{GaN} / \mathrm{Al}_{2} \mathrm{O}_{3}$ [16]. Although this elongation corresponds to the mosaicity reported above, the elongation along $\mathrm{q}_{\mathrm{x}}$ could also originate from in-plane residual microstrains (distribution of the in-plane lattice parameter around the bulk value). To discriminate these two possible effects (mosaicity and microstrain), we have recorded a RSM around the asymmetrical ( $-1-13)$ node of the diamond film (Fig. 5(b)). The node is centered on the bulk value, which confirms that the diamond film is mainly relaxed. It is also elongated with an angle of $\sim 25^{\circ}$ with respect to $\mathrm{q}_{\mathrm{x}}$, corresponding to the angle between $\langle 113\rangle$ and $\langle 001\rangle$ planes, whereas it is narrow along the $q_{x}$ direction. This observation demonstrates that the heteroepitaxial diamond film suffers mainly from a rotational disorder due to its $0.6^{\circ}$ polar mosaicity.

In order to evaluate the in-plane microstrain level on the diamond surface, we perform GIXRD analysis on the (220) reflections with Soller slits of $0.114^{\circ}$. A $2 \theta \chi$ - $\phi$ scan is shown in Fig. 6(a). The FWHM of the peak is about $0.33^{\circ}$ larger than the lateral instrumental resolution (estimated to be $\sim 0.15^{\circ}$ by measuring a diamond single-crystal from element 6 in the same conditions). That means the broadening of the peak in these conditions mainly comes from the distribution of the in-plane lattice parameter (in-plane microstrain) due to structural defects. Taking into account the peak broadening due to the instrumental contribution, the inplane microstrain level at the diamond surface has been evaluated by deconvolution to be about $\pm 0.1 \%$.

It might be interesting to compare this result with the average Raman data recorded at the heteroepitaxial diamond surface in Fig. 2. According to ref. [17,18], the broadening of the Raman line caused by a random compressive and tensile uniaxial stress occurs at a rate of 

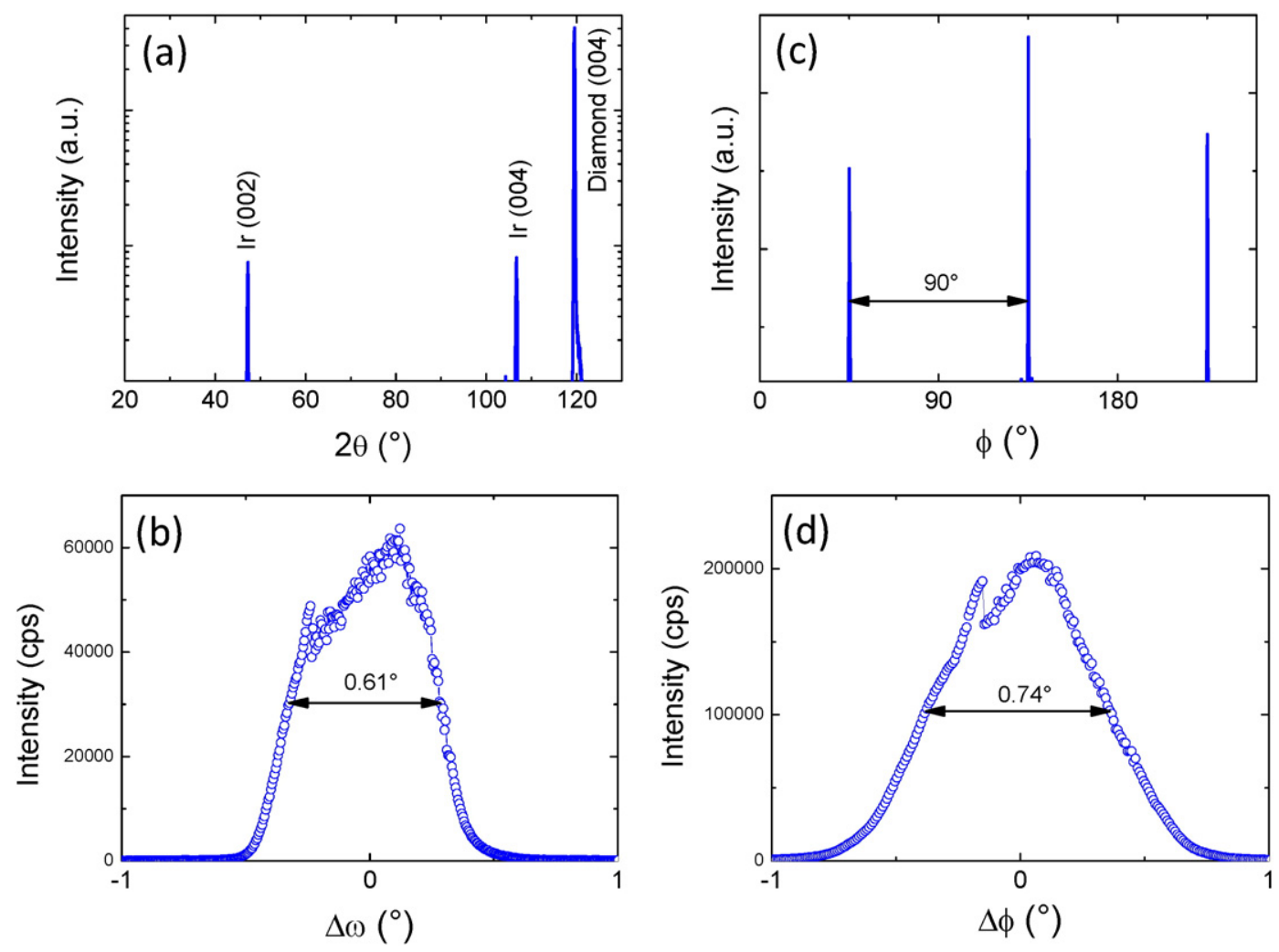

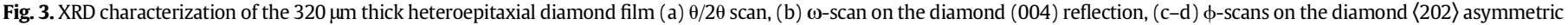
reflections.

$\pm 5 \times 10^{10} \mathrm{dyn} / \mathrm{cm}^{2}$ per FWMH of $\sim 10 \mathrm{~cm}^{-1}$ in diamond. From the typical $3 \mathrm{~cm}^{-1}$ Raman FWHM measured in this work at the surface, the random stress could be evaluated at a level of $\pm 1.5 \times 10^{5} \mathrm{~Pa}$, i.e. a random strain of $\pm 0.13 \%$. This corresponds well to the order of magnitude measured by using GIXRD.

By performing an azimuthal $\phi$-scan in these particular conditions of GIXRD, in which only the first $\sim 100 \mathrm{~nm}$ of the crystal were probed, we could measure the in-plane mosaicity of the crystal close to the final grown surface. For a $320 \mu \mathrm{m}$ thick diamond, we found a strong reduction of the in-plane mosaicity: from $0.74^{\circ}$ averaged on the whole crystal (conventional XRD, Fig. 3(d)) to $0.37^{\circ}$ at the crystal surface (Fig. 6(b)). Here again, these values are close to the state-of-the art for heteroepitaxial diamond films grown on iridium [19], which confirms the high structural quality of our samples. More generally, a mosaicity reduction for thick films have also been reported for heteroepitaxial diamond in both out-of-plane and in-plane directions [11].

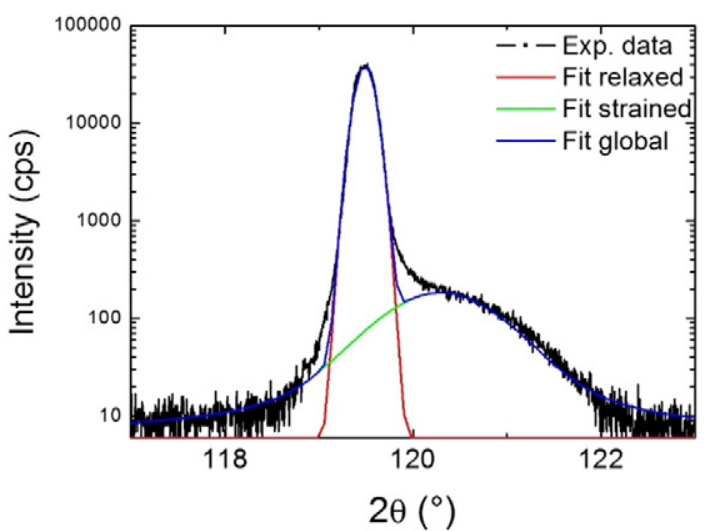

Fig. 4. Zoom of the $\theta / 2 \theta$ scan centered on the diamond (004) reflection (Fig. 3(a)).

\subsection{Observation of dislocations by $C L$}

Different techniques are commonly used to observe dislocations in semiconductors. The most direct, is the transmission electron microscopy (TEM) which allows the indexation of dislocation burger vectors. However, it is limited by the small field of view and also by the preparation of thin lamellae, especially difficult for diamond. X-ray topography is also well suited for the observation and indexation of dislocations but is limited to high quality crystals presenting low dislocation densities [20]. A widely spread technique for the characterization of dislocations consists in revealing the threading dislocations emerging at the final growth surface by a chemical etching leading to etch pits formation and counting them. Alternatively, cathodoluminescence is a convenient non-destructive technique for the observation of dislocations in semiconductors. Dislocations act as non-radiative recombination centers for electron-hole pairs and excitons, therefore they appear with a dark contrast in CL images recorded in the near bandgap region [13,21,22, 23]. CL offers large fields of view and can be performed on both surface and cross section views.

Presented in Fig. 7 (a) and (b) are SEM and CL images of the surface of $200 \mu \mathrm{m}$ thick heteroepitaxial film recorded at low temperature. The SEM image shows macro-steps at the surface, as sometimes observed for thick diamond CVD films grown without nitrogen addition [23]. On the other hand, in the CL images, black spots are observed. They are attributed to single emerging dislocations, while black lines are attributed to emerging dislocations regrouped along lines. In Fig. 8, black points periodically spaced by approximately $2 \mu \mathrm{m}$ reveal the probable presence of parallel dislocations periodically emerging at the surface. This kind of defects is known to accommodate small twist angles between slightly misoriented parts of a crystal. A line with one dislocation by micrometer would correspond to a misorientation of $0.02^{\circ}$ between adjacent crystals [24]. We further observe that the dislocations are arranged in a network of cells with a typical diameter of 5 to $50 \mu \mathrm{m}$, increasing 

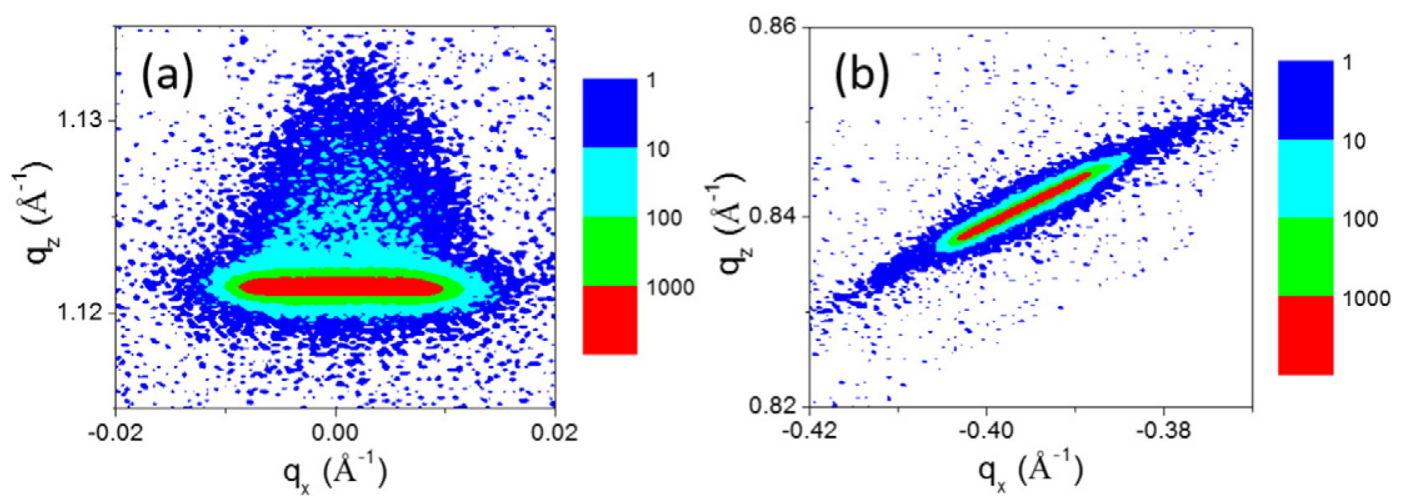

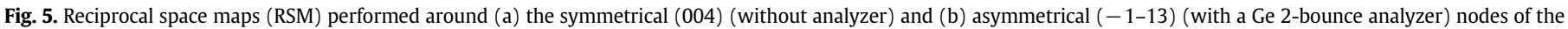
heteroepitaxial diamond film $320 \mu \mathrm{m}$ thick.

with thickness. Such defects, which are likely to be typical of heteroepitaxial diamond, have to be distinguished from the conventional grain boundaries of polycrystalline diamond. M. Schreck et al. have indeed shown using TEM that grain boundaries in heteroepitaxial diamond on iridium are undetectable for samples thicker than a few microns [25].

The dislocation density calculated inside the main cell of Fig. 7 (b) is about $4 \times 10^{6} \mathrm{~cm}^{-2}$. At the cell borders, the dislocation density appears significantly higher, in the range of $10^{8} \mathrm{~cm}^{-2}$ or above. Dislocations at cell borders can be closer than the dislocation resolution of $\sim 1 \mu \mathrm{m}$ in the CL experimental conditions used in this work. Then, an average feature of the threading dislocation density over the whole crystal appears difficult to extract accurately with CL in this sample. A technique with a higher resolution would be required for counting them at the cell border, such as proposed by Ichikawa et al. [26].

To further study the propagation of dislocations during growth, cross sections of the diamond film $200 \mu \mathrm{m}$ thick were prepared. SEM and CL images are reported in Fig. 7 (c) and (d). The surface is smooth after ion beam polishing, as observed from the SEM image with almost no contrast. On the contrary, CL images show black lines which are moreor-less oriented parallel to [001] growth direction. Note that different $\mathrm{CL}$ contrasts are observed for these lines. The low contrast lines could be the result of single dislocations, while the stronger contrast could be related to a group of dislocations, such as observed at the cell borders in plane-view.

As a dislocation is not mobile in diamond even at the high temperatures used for diamond growth, it is indeed expected to propagate by minimizing its energy, proportional to its length. Extending their length from a (001) growth front, as here, the [001] propagation direction is then favoured theoretically. The presence of macrosteps has also been shown to deviate dislocations locally [27]. However, due to the high dislocation density, dislocation interactions are more likely to be the reason for the slight deviations observed from the exact [001] direction.

\subsection{Residual strain evidenced at the microscale by Raman and $C L$}

In Fig. 9 are presented the CL images and the Raman mapping taken on the same area of the cross section, on a $140 \mu \mathrm{m}$ depth from the surface for the $200 \mu \mathrm{m}$ diamond film. The CL image reveals that the network of dislocations evidenced previously forms a columnar structure along [001]. The column diameter tends to increase while the dislocation density decreases during the film growth. The Raman linewidth appears to be directly correlated to the dislocation density, as already shown by C. Stehl et al using an etch pit counting approach. [10].

At the macroscale, the heteroepitaxial diamond films are almost totally relaxed as shown previously by the XRD analysis. However, the width of the diffraction peak in Fig. 6(a) attests the presence of in plane micro-deformations, related to slight variations of the lattice parameter a [28]. Raman mappings in confocal mode provide first insights on the diamond deformations at the local scale. In Fig 9(c) is plotted the Raman peak position extracted by fitting (note that no splitting of the Raman peak could be observed). Interestingly, the peak position of Fig 9(c) brings different information than the linewidth of Fig 9(b). Strong deviations are observed above (up to $-0.9 \mathrm{~cm}^{-1}$ ) and below (up to $\left.+0.7 \mathrm{~cm}^{-1}\right)$ the relaxed Raman peak frequency $\left(1332.5 \mathrm{~cm}^{-1}\right)$ [29]. The fact that the local deviations observed in Fig 9 (c) are almost symmetrical around the relaxed position is likely to explain why the sample was found relaxed at the macro-scale in XRD. Without being quantitative neither on the intensity nor on the direction of residual strain, Raman mapping clearly evidences a local deformation, probably induced here by the strain-field of dislocations. While at the macroscale, $\mathrm{XRD}$ indicates the crystal is, in average, relaxed to its usual lattice
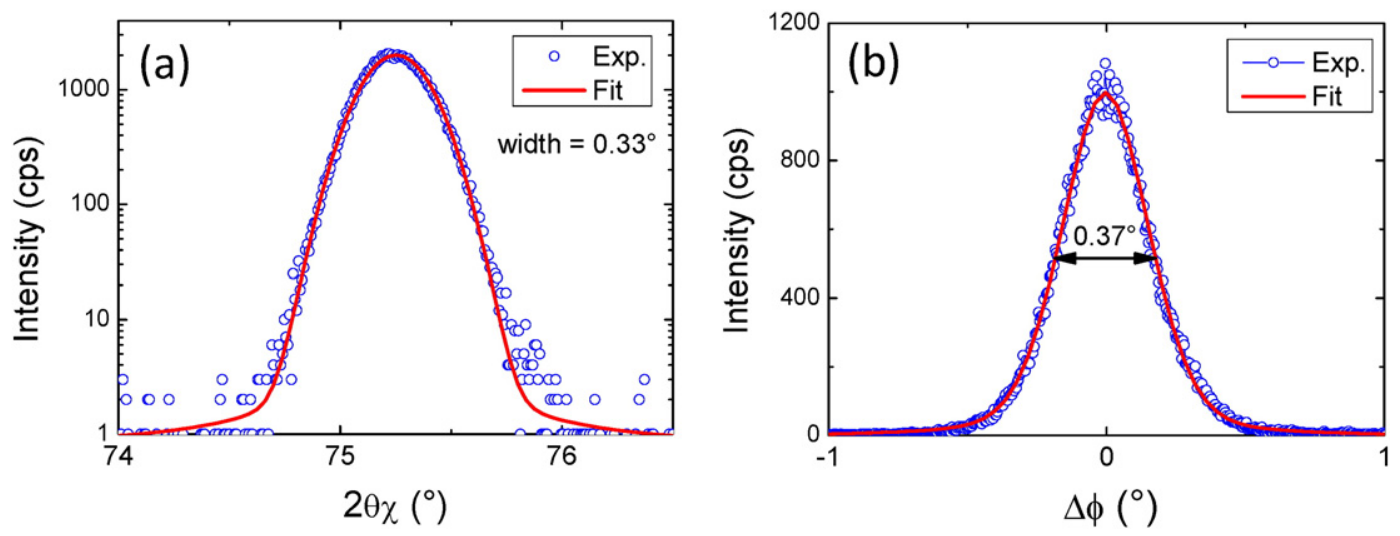

Fig. 6. GIXRD around the (220) reflection of the diamond surface. (a) $2 \theta \chi-\phi$ scan and (b) $\phi$ scan. 

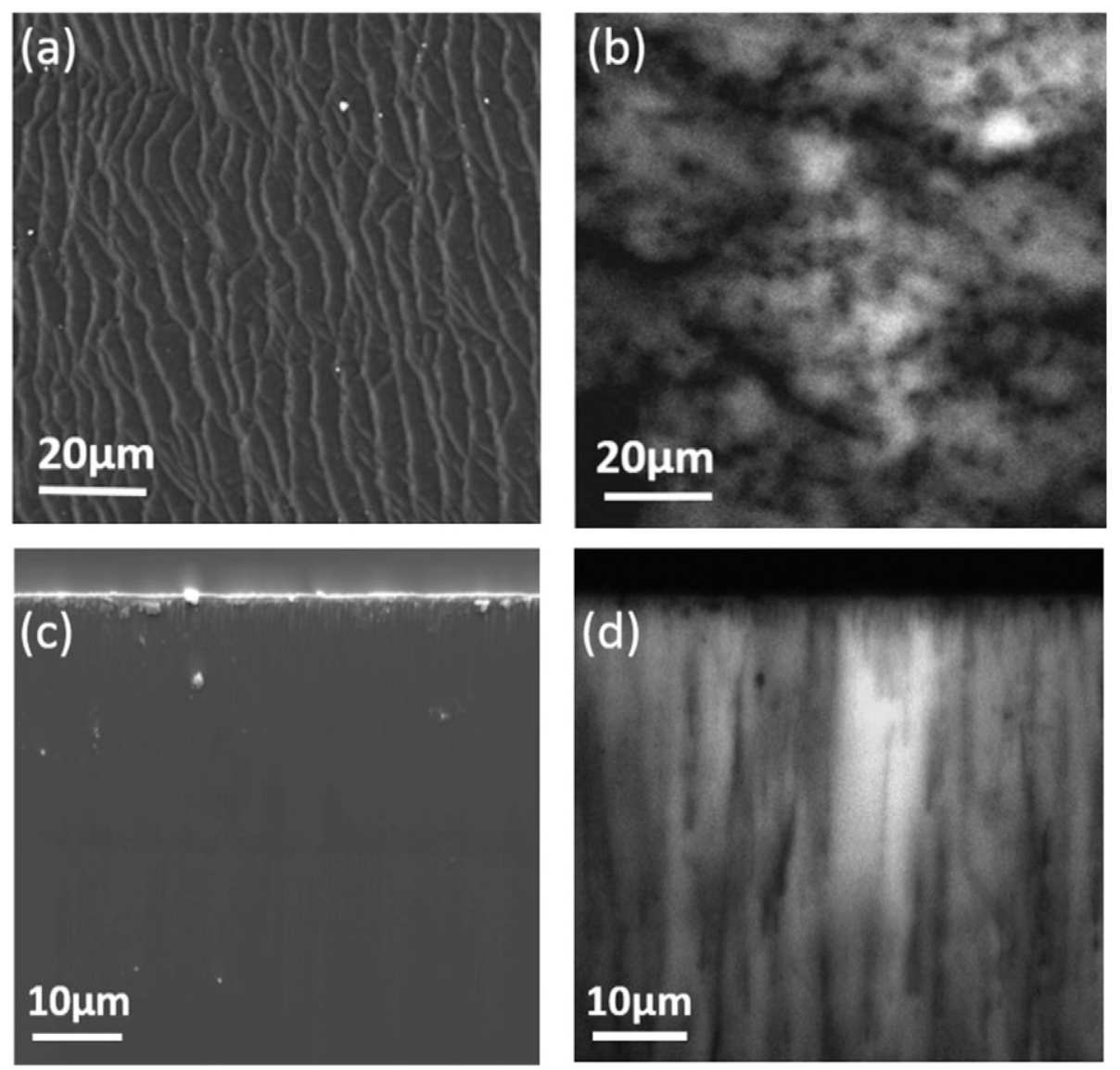

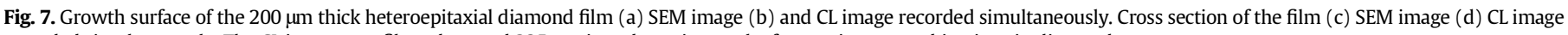
recorded simultaneously. The $\mathrm{CL}$ images are filtered around $235 \mathrm{~nm}$ in order to image the free exciton recombinations in diamond.

parameter, mapping of the Raman frequency shows that residual strain remains important and inhomogeneous at the micrometer scale.

A set of CL spectra which are typically observed from the heteroepitaxial diamond samples is also presented in Fig. 10, plotted in the UV region of exciton recombinations. These high resolution spectra were recorded with the electron beam focused at different locations on the surface of the $200 \mu \mathrm{m}$ thick heteroepitaxial diamond. The reference spectrum of an "electronic grade" single crystal (Element 6) is also presented for comparison in (a). From this reference, the TO-, TAand LO-phonon-assisted recombinations of free excitons are identified at their energy of 5.27, 5.32 and $5.24 \mathrm{eV}$ respectively, as commonly

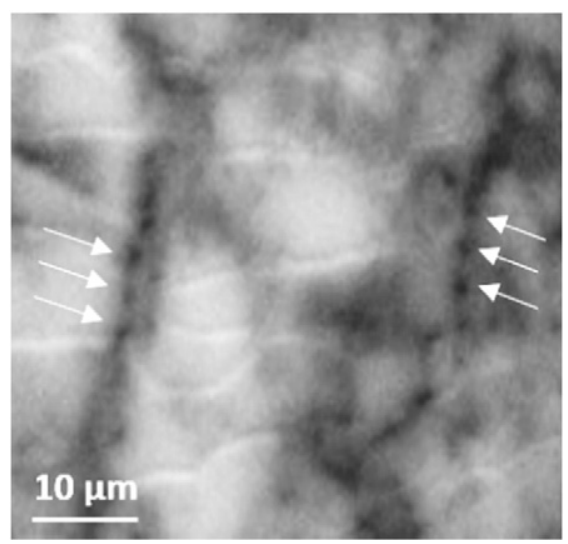

Fig. 8. CL image of exciton recombinations from the $320 \mu \mathrm{m}$ thick heteroepitaxial diamond film seen from the top. The white arrows indicate dislocations emerging almost periodically along a line. Note that the white contrast is a geometrical effect due to the presence of macrosteps. observed in unstrained diamond. In Fig. 10 (b) (c) (d) (e), dramatic changes are observed for the free exciton recombinations occurring in heteroepitaxial diamond: the free exciton recombinations appear shifted and splitted compared to the reference single crystal.

As described previously, the effect of strain on phonon mode frequencies is weak. The typical shift observed in heteroepitaxial diamond with Raman spectroscopy is $\sim 1 \mathrm{~cm}^{-1}$ over $1332 \mathrm{~cm}^{-1}$. In energy, it corresponds to $\sim 0.1 \mathrm{meV}$ over $165 \mathrm{meV}$. The spectral resolution of CL spectra in the UV range being typically $1 \mathrm{meV}$, the phonon energy involved in exciton recombinations can be safely considered as independent on strain. This allows us to identify two groups of TO and TA recombinations which are separated by a constant value of $54 \mathrm{meV}$. This value accurately matches the energy difference between TO and TA phonons involved in the free exciton recombinations in diamond [30]. The two groups of TO and TA recombinations are indicated by using brackets in spectra of Fig. 10.

Considering the group of TO-assisted recombinations, its maximum is systematically shifted at lower energies compared to the reference homoepitaxial CVD diamond. Concomitantly with the low-energy shift, the splitting between TO lines increases: up to $41 \mathrm{meV}$ is measured between the low and high energy component in the group of TOassisted recombinations. The low energy threshold for the recombination of free excitons is $h v=E_{g}-E_{X}-\hbar \omega$, where $\hbar \omega$ is the phonon energy, $E_{X}$ the exciton binding energy and $E_{g}$ the bandgap energy. As $\hbar \omega$ and $E_{X}$ can be both considered as independent on strain effects, the shift and splitting of the free exciton recombinations are first evidences of a change in the diamond electronic structure. We attribute this change to the strain field which appears strongly non uniform at the micrometer scale in heteroepitaxial diamond films.

It is well-known that the band structure of a semiconductor can be deeply modified under strain. One of the most famous applications is 

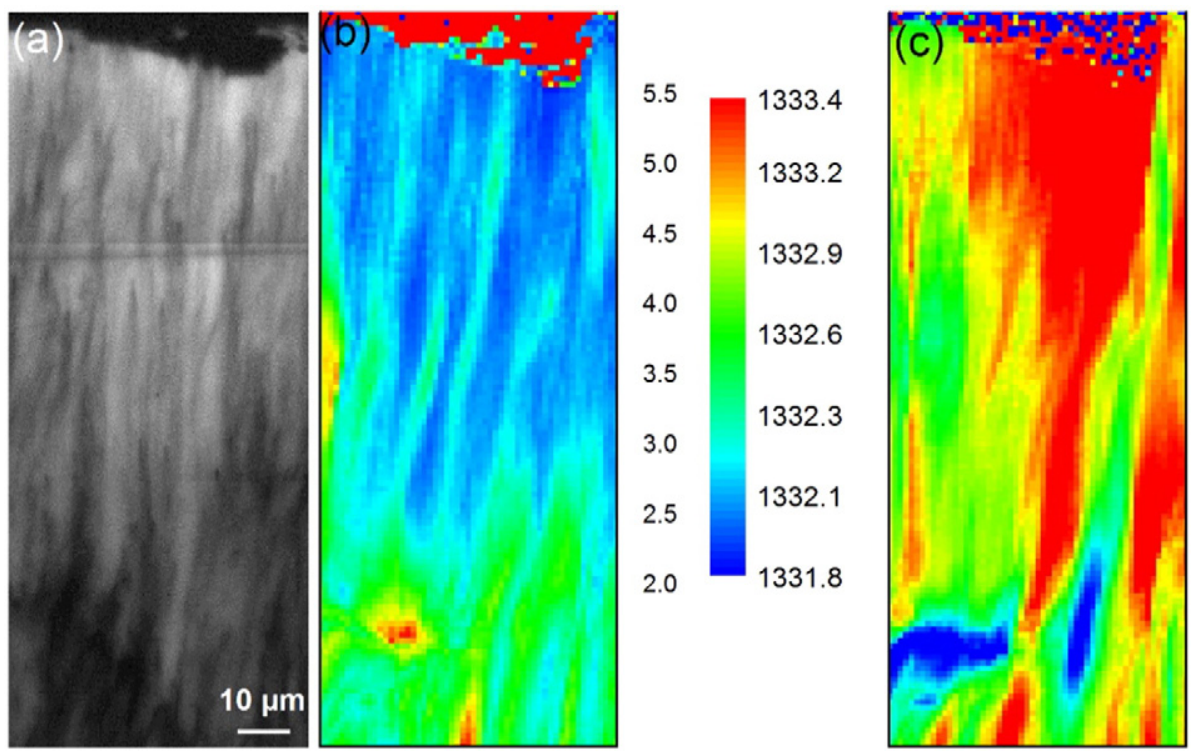

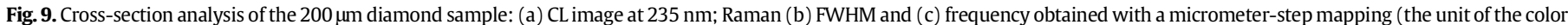
scale bar is $\mathrm{cm}^{-1}$ ). The vertical direction correspond to the [001] growth axis.

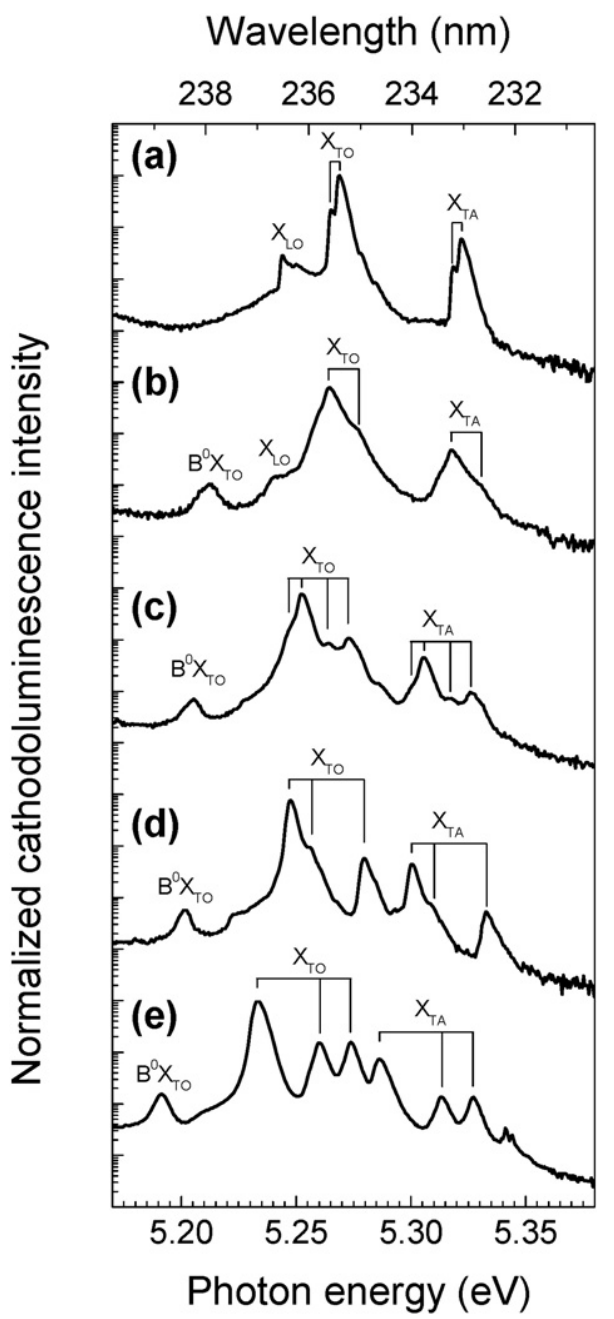

Fig. 10. High resolution CL spectra of exciton recombinations in diamond at $T=10 \mathrm{~K}$ : (a) reference single crystal, (b), (c), (d) and (e) heteroepitaxial diamond grown on iridium when the beam is focused at different locations on the diamond surface. The spectra are normalized to their maximum intensity and plotted in a logarithm scale. the high mobility transistors obtained with a strained channel. The strain effect is well documented in silicon [31]. The different behavior of the conduction and valence bands under strain can lead to a large variety of situations, depending on directions and amplitudes of the strain field. For instance, it can lift the conduction band degeneracy and/or reverse the light and heavy hole valence bands. A hydrostatic pressure usually induces a shift of the bandgap, while anisotropic strain fields are responsible for a splitting of the valence and/or conduction bands.

In heteroepitaxial diamond, depending on the position on the sample, we observe from 2 to 4 lines for each phonon-assisted recombination. These lines are shifted but also splitted compared to their positions in the reference single crystal. They might correspond to the modifications of the diamond band structure under an anisotropic strain.

The presence of a slight boron contamination in the order of $\sim 1 \times 10^{14}$ atoms $/ \mathrm{cm}^{3}$ can also be inferred from the observation of boron-bound exciton recombinations [32], labelled $\mathrm{B}^{0} \mathrm{X}_{\mathrm{TO}}$ in the spectra of Fig. 10. Its recombination energy is maximum at $5.21 \mathrm{eV}$ in relaxed homoepitaxial diamond. In heteroepitaxial diamond, it is detected at lower energies, with a similar shift than observed for the maximum of $\mathrm{X}_{\mathrm{TO}}$. This is also coherent with a modification of the diamond electronic structure under strain.

As a summary, the exciton recombination energies at low temperature reveal a high sensitivity to the residual strain present at the microscale in heteroepitaxial diamond. Being clear that the dislocation reduction and the strain management will be important issues for upscaling the growth process, the luminescence spectroscopy already appears as a key characterization of heteroepitaxial diamond. To further assess the strain quantitatively from luminescence spectra, the values of the so-called deformation potentials would be necessary. While they are well-known for most semiconductors, a strong discrepancy among their theoretical values is observed for diamond [33]. The full set of diamond deformation potentials basically remains to measure at the present time.

\section{Conclusions}

The crystalline structure of heteroepitaxial diamond films grown on iridium was investigated in details using a multi-scale approach with a combination of Raman, XRD and CL techniques. The linewidth of the first order diamond peak measured in Raman is comparable to values 
reported in literature for heteroepitaxial diamond films thicker than $100 \mu \mathrm{m}$. XRD spectra confirm the expected epitaxial relationship $\langle 100\rangle$ diamond $(001) / /\langle 100\rangle$ Iridium $(001) / /\langle 100\rangle \mathrm{SrTiO}_{3}(001)$. Polar and azimuthal crystalline mosaicities equal to $0.61^{\circ}$ and $0.74^{\circ}$, respectively, are at the state-of-the-art for comparable thicknesses. Using the more surface sensitive GIXRD configuration, the azimuthal mosaicity is reduced down to $0.37^{\circ}$ indicating an improvement of the crystalline quality away from the substrate interface (i.e. for thicker films). The $\theta / 2 \theta$ scan performed on diamond (004) reflection shows that only $3 \%$ of the diamond film volume presents a slightly smaller vertical (c) lattice parameter of $\sim 0.5 \%$ than relaxed diamond.

Whereas the diamond lattice is found mainly relaxed macroscopically, in-plane microstrains of $\pm 0.1 \%$ at the diamond surface has been revealed by GIXRD and the combined CL/Raman mapping observations performed on a cross-section reveal deformations of the diamond lattice at the micrometer scale. All these analytical methods converge to evidence the presence of microstrains in heteroepitaxial diamond. Such microstrains - probably due to the network of dislocations revealed by $\mathrm{CL}$ images - induce a shift and a splitting of free exciton recombinations at low temperature. The splitting indicates the local strain is anisotropic but the values of the deformation potentials would be needed to further evaluate the local modifications of the diamond electronic structure.

Our current developments concern the growth of heteroepitaxial diamond films on up-scalable $\mathrm{Ir} / \mathrm{SrTiO}_{3} / \mathrm{Si}(001)$. These new substrates appear very promising to achieve diamond wafers usable for electronics or radiation detection applications.

\section{Prime novelty statement}

The current manuscript provides a detailed analysis of the crystalline structure and the structural defects in heteroepitaxial diamond films grown on iridium using a multi-scale approach with a combination of Raman, XRD and CL techniques.

\section{Acknowledgments}

The authors acknowledge the French National Research Agency for financial support of the HIRIS project (HIRIS-ANR-12-BS09-0001-01) and C. Vilar for cross section preparations and SEM-CL maintenance.

\section{References}

[1] M. Schreck, J. Asmussen, S. Shikata, J.C. Arnault, N. Fujimori, Large-area high-quality single crystal diamond, MRS Bull. 39 (2014) 504-510.

[2] H. Yamada, A. Chayahara, H. Umezawa, N. Tsubouchi, Y. Mokuno, S. Shikata, Fabrication and fundamental characterizations of tiles clones of single-crystal diamond with 1-inch size, Diam. Relat. Mater. 24 (2012) 29-33.

[3] H. Kawashima, H. Noguchi, T. Matsumoto, H. Kato, M. Ogura, T. Makino, S. Shirai, D. Takeuchi, S. Yamasaki, Electronic properties of diamond Schottky barrier diodes fabricated on silicon-based heteroepitaxially grown diamond substrates, Appl. Phys. Express 8 (2015) 104103

[4] A. Chavanne, J. Barjon, B. Vilquin, J. Arabski, J.C. Arnault, Surface investigations on different nucleation pathways for diamond heteroepitaxial growth on iridium, Diam. Relat. Mater. 22 (2012) 52-58

[5] J.C. Arnault, Highly oriented diamond films on hetero-substrates: current state of the art and remaining challenges, Surf. Rev. Lett. 10 (2003) 127-146.
[6] B.R. Stoner, J.T. Glass, Textured diamond growth on (100) beta-SiC via microwave plasma chemical vapor deposition, Appl. Phys. Lett. 60 (1992) 698-700.

[7] J.C. Arnault, S. Delclos, S. Saada, N. Tranchant, P. Bergonzo, Stability of 3C-SiC surfaces under diamond growth conditions, J. Appl. Phys. 101 (2007) 14904-14910.

[8] N. Vaissiere, S. Saada, M. Bouttemy, A. Etcheberry, P. Bergonzo, J.C. Arnault, Heteroepitaxial diamond on iridium: new insights on domain formation, Diam. Relat. Mater. 36 (2013) 16-25.

[9] S. Gsell, T. Bauer, J. Goldfuß, M. Schreck, B. Stritzker, A route to diamond wafers by epitaxial deposition on silicon via iridium/yttria-stabilized zirconia buffer layers, Appl. Phys. Lett. 84 (2004) 4541-4543.

[10] C. Stehl, M. Fischer, S. Gsell, E. Berdermann, M.S. Rahman, M. Traeger, O. Klein, M. Schreck, Efficiency of dislocation density reduction during heteroepitaxial growth of diamond for detector applications, Appl. Phys. Lett. 103 (2013) 151905.

[11] M. Fischer, S. Gsell, M. Schreck, A. Bergmaier, Growth sector dependence and mechanism of stress formation in epitaxial diamond growth, Appl. Phys. Lett. 100 (2012) 041906.

[12] E. Berdermann, M. Pomorski, W. de Boer, M. Ciobanu, S. Dunst, C. Grah, M. Kiš, W. Koenig, W. Lange, W. Lohmann, R. Lovrinčić, P. Moritz, J. Morse, S. Mueller, A. Pucci, M. Schreck, S. Rahman, M. Träger, Diamond detectors for hadron physics research, Diam. Relat. Mater. 19 (2010) 358-367.

[13] A. Tallaire, J. Barjon, O. Brinza, J. Achard, F. Silva, V. Mille, R. Issaoui, A. Tardieu, A. Gicquel, Diam. Relat. Mater. 20 (2011) 875-881.

[14] M. Schreck, H. Roll, J. Michler, E. Blank, B. Stritzker, Stress distribution in thin heteroepitaxial diamond films on Ir/SrTiO3 studied by x-ray diffraction, Raman spectroscopy, and finite element simulations, J. Appl. Phys. 88 (2000) 2456.

[15] S. Gsell, M. Schreck, B. Stritzker, Crystal tilting of diamond heteroepitaxially grown on vicinal Ir/SrTiO3 (001), J. Appl. Phys. Vol. 96 (2004) 1413.

[16] S. Pereira, M.R. Correia, E. Pereira, K.P. O'Donnell, E. Alves, A.D. Sequeira, N. Franco, I.M. Watson, C.J. Deatcher, Strain and composition distributions in wurtzite InGaN/ GaN layers extracted from x-ray reciprocal space mapping, Appl. Phys. Lett. Vol. 80 (2002) 3913

[17] J. Wagner, C. Wild, W. Miller-Sebert, P. Koidl, Appl. Phys. Lett. 61 (1992) 1284.

[18] M. Yoshikawa, G. Katagiri, H. Ishida, A. Ishitani, M. Ono, K. Matsumura, Appl. Phys. Lett. 55 (1989) 2608.

[19] M. Fischer, S. Gsell, M. Schreck, R. Brescia, B. Stritzker, Preparation of 4-inch Ir/YSZ/Si (001) substrates for the large-area deposition of single-crystal diamond, Diam. Relat. Mater. 17 (2008) 1035-1038.

[20] M.P. Gaukroger, P.M. Martineau, M.J. Crowder, I. Friel, S.D. Williams, D.J. Twitchen, $\mathrm{X}$-ray topography studies of dislocations in single crystal CVD diamond, Diam. Relat. Mater. 17 (2008) 262-269.

[21] E. Meissner, S. Schweigard, J. Friedrich, T. Paskova, K. Udwary, G. Leibiger, F. Habel, Cathodoluminescence imaging for the determination of dislocation density in differently doped HVPE GaN, J. Cryst. Growth 340 (2012) 78-82.

[22] T. Sekiguchi, K. Sumino, Cathodoluminescence study on dislocations in silicon, J. Appl. Phys. 79 (1996) 3253.

[23] A. Tallaire, J. Achard, F. Silva, O. Brinza, A. Gicquel, Growth of large size diamond single crystals by plasma assisted chemical vapour deposition: Recent achievements and remaining challenges, Comptes Rendus Physique Vol. 14 (2013) 169.

[24] M.G. Tsoutsouva, V.A. Oliveira, D. Camel, J. Baruchel, B. Marie, T.A. Lafford, Mono-like silicon ingots grown on low angle misoriented seeds: Defect characterization by synchrotron X-ray diffraction imaging, Acta Mater. 88 (2015) 112-120.

[25] M. Schreck, CVD Diamond for Electronic Devices and Sensors, John Wiley \& Sons Ltd, 2009

[26] K. Ichikawa, H. Kodama, K. Suzuki, A. Sawabe, Thin Solid Films 600 (2016) 142

[27] P. Martineau, M. Gaukroger, R. Khan, D. Evans, Effect of steps on dislocations in CVD diamond grown on $\{001\}$ substrates, Phys. Status Solidi C 8 (2009) 1953-1957.

[28] T. Ungár, Dislocation densities, arrangements and character from X-ray diffraction experiments, Mater. Sci. Eng. A 309-310 (2001) 14-22.

[29] A.M. Zaitsev, Optical Properties of Diamond: A Data Handbook, University of Bochum, Germany, Springer, Berlin, 2001.

[30] P.J. Dean, Bound excitons and donor acceptor pairs in natural and synthetis diamond, Phys. Rev. 139 (1965) A588.

[31] Yongke Sun, Scott.E. Thompson, Toshikazu Nishida, Strain Effect in Semiconductors Theory and Device Applications, Springer, 2010.

[32] J. Barjon, T. Tillocher, N. Habka, O. Brinza, J. Achard, R. Issaoui, F. Silva, C. Mer, P. Bergonzo, Boron acceptor concentration in diamond from excitonic recombination intensities, Phys. Rev. B 83 (2011) 073201.

[33] S. Adachi, Handbook on Physical Properties of Semiconductors I, Group-IV Semiconductors, Kluwer Academic Publishers, 2004. 